\title{
Discussion to papers of Dr Watson and Dr Sutton et al.
}

\section{Dr HARDY (Chairman)}

DR Pool (Holland). It is always a pleasure to hear Watson read a paper, it's the language and he is a good clinician and I'd like to congratulate him that he kept the surgeon out of doors, because in these cases I should have asked the surgeon just to open the door, otherwise we can't see what is in it. But he managed to do very good work on those two patients.

Dr Yeo (Australia). Mr Chairman I'd just like to ask a question. I'm not convinced that what we are looking at in part isn't the mesenteric artery syndrome that many orthopaedic surgeons see in the extension plaster. We've had two cases like this late onset and by simply posturing the patient we can relieve the gastric distension. There is no doubt that some of the signs and symptoms described by our two speakers are associated with autonomic dysreflexia which you would expect with the distended stomach but my comment and question is along the lines that I wonder whether we couldn't approach the treatment in these three patients perhaps with simply posturing the patient so that they are no longer lying in that hyperextended position with the mesenteric artery in the mesentery causing obstruction in the region of the duodenum.

Dr MEINECKe (Germany). I agree with John Yeo. I have a feeling that the cases of Norman Watson are not incidentally related to the spinal cord injury; the time between the occurrence of the distension is too long. I believe the so-called ileus after a spinal cord injury is not an ileus, it is an atonic bowel but I do feel that probably these two cases relate to the vascular system, thrombosis or something like this.

Mr Nuseibeh (G.B.). May I ask Dr Watson if he had done blood cultures. I say that because I believe that the paraplegic is exposed to frequent urinary infection and bacteraemia and septicaemia. We notice in every flare-up there is always sluggishness in the bowel and if the attack is severe late ileus can develop.

DR WATSON. The episodes that occurred in the second patient were so dramatic and they were switched on literally within minutes. One minute this man was perfectly well eating his dinner and half-an-hour later he was at death's door with the intense tachycardia, clamminess, sweating, temperature and abdominal distension. Then, when the episode resolved 3 days later there was again a very dramatic resolution which occurred within minutes. Half-an-hour after the man telling me that he felt he was going to die he could eat steak and chips, he suddenly felt so much better, and I feel that this can only be a neurological switching on and switching off of sympathetic overactivity. I quite agree that they started with temperature, we did blood cultures in the second one and we did a pyelogram. Indeed we were looking for a urinary tract problem, and his pyelogram in the middle of the third episode was exactly normal and the blood cultures were normal, so I don't think it is a urinary infection. I admit that it started off with temperature and I'm just wondering just exactly whether an infection was responsible, that's the only thing I can think of to trigger it off, but it wouldn't be responsible for the on-off situation which occurred over 6 weeks, with six separate episodes, and between those episodes the man was perfectly well and normal. Dr Yeo, I've no experience of this but I had a surgeon in attendance at these cases. In fact the surgeon was quite bewildered, a very experienced gastro-intestinal surgeon in Sheffield who was involved in the management of these cases and he had seen nothing like this ever before, and he was completely bamboozled. So that I don't think it is a thing which is commonly found in surgical wards. I think it is some form of sympathetic overactivity, what triggers it off. I don't know but it is very alarming and indeed it was so alarming that the man himself felt he was going to die, each time he had this episode he felt very ill and he looked it, he looked dreadful and indeed I had a new car that month and when I got the telephone call 
that night I came out of the drive so quickly that I ran into a parked car double parked on the other side of the road. The emergency was such that we were very very worried and agitated about it, and everything suddenly got better. I'm just mentioning it to the Society just in case they get a similar problem that I'll know how to deal with it in future.

Dr MCCluRgo (G.B.). We have had a 75-year-old man who presented a similar picture to Dr Watson's case. He is unfortunately dead now. He died from a subarachnoid haemorrhage. He presented with abdominal distension, vomiting, sweating. In fact on two occasions he had laparotomies done by a past President of the Royal College of Surgeons. He had had barium meals and all these investigations and he was definitely to us a case of autonomic dysreflexia and nothing was found. He was a $\mathrm{C}_{5} / \mathrm{C} 6$ level.

DR MCSWEENEY (G.B.). I think the mechanical ileus, the ileus associated with the superior mesenteric vessel of the ligament of Trietz is a slow onset and associated with a pillow under the lumbar spine and the inference is fairly obvious if you take out the pillow you are over the crisis, quite a different picture to the dramatic ileus described by Sutton and Norval Watson.

DR HARDY. Thank you. 